\title{
Rethinking the war on cancer: multidisciplinary collaborations between biologists and physical scientists
}
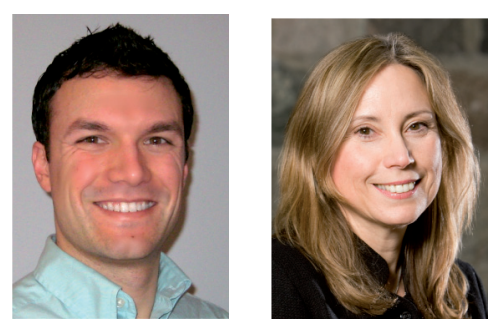

"...to take full advantage of the

more integrative approaches, what is needed is an evolution of

the philosophy - not just the technology - of cancer research."

Devin T Rosenthal' \& Sofia D Merajver*

Tufts University, 75 Kneeland St., 14th floor, Boston, MA 02111, USA

*Author for correspondence: University of Michigan, 1500 E Medical Center Dr., 7127 CCGC, Ann Arbor,

Ml 48109, USA = Tel.: +1 7349366884 = Fax: +1 7349367376 m smerajve@med.umich.edu

Ever since the discoveries of tumor suppressors and oncogenes in the mid-20th century, the study of cancer has been the study of the genes underlying the disease. The strategy has been to understand genomic copy number and sequence alterations in tumor genomes and assume that effective cancer therapies would then arise from this knowledge.

An impressively large and diverse body of work has been devoted to identifying and characterizing individual genes that are altered in cancers. This research has led to a detailed understanding of the mutations and genomics of many oncogenes and tumor suppressors. It has also revealed how relatively minor perturbations, such as single nucleotide polymorphisms, can impact protein expression and function, and ultimately even patient survival [1]. This approach has yielded successful targeted therapies such as trastuzumab and Gleevec ${ }^{\circledR}$ (Novartis, Basel, Switzerland) $[2,3]$; however, these few gains stand in sharp contrast to the large number of gene-centered findings that have not translated into useful therapies.

The reductionist single-gene approach has yielded much of what we know today about gene and protein functions and pathways, but is very limiting when it comes to integration. We now understand that cancer is a highly complex disease that involves more than just a single gene or a handful of genes acting in a single cell. Gene regulatory networks within cancer cells are highly interconnected and redundant; cancer cells influence, and are influenced by, numerous components of their microenvironment, both cellular and acellular, and the cells comprising an individual cancer are themselves highly heterogeneous - phenotypically and genetically. Next-generation sequencing is a major step towards addressing the genetic complexity of cancer; however, to take full advantage of the more integrative approaches, what is needed is an evolution of the philosophy - not just the technology - of cancer research.

We understand that cancer is highly adaptable and constantly evolving. Tumors will eventually develop resistance to therapies. Metastasizing cancer cells adapt to ever-changing microenvironment conditions and colonize many new organs as the disease progresses. This plasticity likely involves dozens to hundreds to thousands of genes and it follows that, rather than individual genes, cellular behaviors (such as proliferation, motility and invasion) are most significant in cancer progression. We therefore propose that cancer should be viewed as a composite of these requisite behaviors - each of which is a dynamic mosaic of gene expression - in order to account for, and ultimately discern, the genes governing these behaviors.

A major problem for biologists undertaking such an integrated approach is the need for ongoing collaborative work with scientists of other disciplines. For example, addressing cell motility during metastasis is more than just a cell biology question. The mechanical properties of cell structures (such as the cytoskeleton, nucleus and membrane), the physical interactions between the cell and the microenvironment, and the abundance and consumption of microenvironmental resources (such as oxygen and glucose) are among the diverse features that factor into, and indeed determine, the characteristics of cell motility. Accordingly, cell motility is best addressed through diverse disciplines that can explore in depth the individual phenomena that contribute to motility.

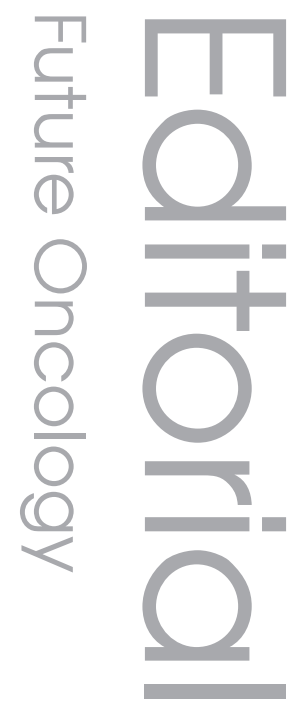

\section{Keywords}

- breast cancer

- collaboration m metastasis

- multidisciplinary $=$ p38 MAPK

\section{Future Medicine part of}


In recent work investigating the function of p38 $\gamma$ in breast cancer cell motility and metastasis [4], we reached a point where our standard approach - cellular and molecular biology - could no longer answer the questions raised by the data we were obtaining. We observed a striking phenotype following p38 $\gamma$ knockdown: drastically impaired cell motility and altered cytoskeletal architecture. We questioned both how these features were related to each other and how they contributed to motion; however, we could not further interrogate this phenotype using conventional cell biology approaches. Instead, we hypothesized that the observed motility phenotype was, at its core, due to mechanical integration of signaling pathways and of external stimuli; namely, that the altered cytoskeletal architecture mediated by p38 $\gamma$ knockdown was generating the observed motility phenotype. Because the root of this question was no longer cell biological, but rather mechanical, we enlisted the collaboration of two mechanical engineering groups to generate a mechanics-based computational model of cell motility in which we could test the effects of modified cytoskeletal architecture on breast cancer cell motility. If it were not for the mechanical models, we would not have been able to test hypotheses related to how cytoskeletal architecture modifies motility.

"If we are to conquer a disease as
complex as cancer we must become as
adaptable and opportunistic as the
disease itself."

The computational models revealed that $\mathrm{p} 38 \gamma$ mediated changes in cytoskeletal architecture were sufficient to alter breast cancer cell motility in both the qualitative and quantitative manners observed in live cells. Interestingly, this link was established in the absence of any genetics (other than p38 $\gamma$ knockdown) and was instead simply based on the physical properties of the motile cell: membrane and actin cytoskeleton rigidity, rates of protrusion and retraction, and other features. This work thus represents an important paradigm that supports integration and contrasts with the traditional 'mechanistic' study. Rather than focusing on cellular signaling mechanisms, many of which can be compensated for by alternate signaling pathways, we focused on the physical and behavioral end point of these signaling networks; in essence accounting for all possible signaling inputs without studying each one in detail - an insurmountable obstacle in itself.
Although such a study does not immediately reveal specific druggable targets, it does provide a systems-wide perspective on aberrant cell behavior (in this example, altered cell motility as a consequence of genetic manipulation) that can ultimately be used to identify new drug targets. Using computational mechanical modeling enabled us to take a top-down approach. By understanding the basic physical properties that contribute to a given cellular behavior (in this instance, cytoskeletal architecture contributing to mesenchymallike cell motility) we could work backwards; first identifying broad signaling networks that contribute to the physical behavior (i.e., cytoskeletal remodelers), narrowing that down to protein families that govern the detailed physical properties underlying the cellular phenotype (i.e., Rho GTPases and stress fiber formation) and finally delineating the specific signaling molecule(s) driving the observed phenotype (in this case, RhoC GTPase). Such an approach addresses issues such as crosstalk and compensation early on by implicitly incorporating them into the original mechanical model. Using signaling-based mathematical modeling and systems biology, one could also aim to predict potential sources of compensation when therapeutically targeting the identified molecular driver (i.e., other Rho GTPases compensating for RhoC knockdown). This approach could eventually determine optimal multi-target therapies aimed at disrupting a behavior, rather than a specific protein.

Other such examples have emerged in recent years, including engineering-based biophysical studies of cell mechanics during motility [5], mathematical models of cancer cell (de)differentiation [6] and the effects of selective pressures on tumor outgrowth [7]. Each of these studies has revealed new biological insights that would be unattainable without interdisciplinary collaboration.

Importantly, these interactions are not unidirectional. In our p38 $\gamma$ study and the other studies referenced here, there is incredible synergy between the results and hypotheses generated by each discipline. Our original cell biology discoveries served as the foundation for the mechanical computational modeling area of investigation. This flow of data was quickly reciprocated, as the discoveries from the models revealed a new leading-edge behavior that we then investigated in live cells. Such synergy and reciprocity is fertile ground for developing and testing new, unexplored concepts regarding cancer behavior, development and progression. If we are to conquer a disease as complex as cancer we must become as adaptable and opportunistic as the disease 
itself. Interdisciplinary research must become the new standard discipline of cancer research. Academic leaders, faculties and students should work together to understand and remove all the very real barriers to this type of work, beginning with how to access funding mechanisms as a group, indirect cost flows, standards for promotion, authorship practices and communication styles, to name just a few. We surmise that the rewards of understanding and controlling cancer will make this small effort worthwhile.

\section{References}

Papers of special note have been highlighted as:

- of interest

1. Riaz M, Berns EM, Sieuwerts AM et al. Correlation of breast cancer susceptibility loci with patient characteristics, metastasis-free survival, and mRNA expression of the nearest genes. Breast Cancer Res. Treat. doi:10.1007/ s10549-011-1663-3 (2011) (Epub ahead of print).

2. Druker BJ, Tamura S, Buchdunger E et al. Effects of a selective inhibitor of the $\mathrm{Abl}$ tyrosine kinase on the growth of Bcr-Abl positive cells. Nat. Med. 2(5), 561-566 (1996).

3. Vogel CL, Cobleigh MA, Tripathy D et al. Efficacy and safety of trastuzumab as a single

\section{Financial \& competing interests disclosure}

The authors have no relevant affiliations or financial involvement with any organization or entity with a financial interest in or financial conflict with the subject matter or materials discussed in the manuscript. This includes employment, consultancies, honoraria, stock ownership or options, expert testimony, grants or patents received or pending, or royalties.

No writing assistance was utilized in the production of this manuscript.

agent in first-line treatment of HER2overexpressing metastatic breast cancer. J. Clin. Oncol. 20(3), 719-726 (2002).

4. Rosenthal DT, Iyer H, Escudero $\mathrm{S}$ et al. p38 $\gamma$ promotes breast cancer cell motility and metastasis through regulation of $\mathrm{RhoC}$ GTPase, cytoskeletal architecture, and a novel leading edge behavior. Cancer Res. 71(20), 6338-6349 (2011).

- Experimental discovery of novel features of cancer cell motility are reproduced by mechanical modeling of the cell motion, which utilizes only the data corresponding to the cytoskeletal architecture.

5. Fu J, Wang YK, Yang MT et al. Mechanical regulation of cell function with geometrically modulated elastomeric substrates. Nat. Methods 7(9), 733-736 (2010).

- Construction and utilization of a polymer-based micropost device opens doors to studying subcellular traction forces during cell movement.

6. Gupta PB, Fillmore CM, Jiang G et al. Stochastic state transitions give rise to phenotypic equilibrium in populations of cancer cells. Cell 146(4), 633-644 (2011).

7. Anderson AR, Weaver AM, Cummings PT, Quaranta V. Tumor morphology and phenotypic evolution driven by selective pressure from the microenvironment. Cell 127(5), 905-915 (2006). 